\section{A48 THE TRAF6 BINDING MOLECULE P62/S0STM1 IS A CRITICAL REGULATOR OF INFLAMMATORY BONE DESTRUCTION}

A Korb, ${ }^{1}$ A Hillmann, ${ }^{1}$ S Bürgis, ${ }^{2}$ G Kollias, ${ }^{3}$ S Hermann, ${ }^{4}$ M Schäfers, ${ }^{4}$ T Pap, ${ }^{5}$ T Weide, ${ }^{1}$ A Gessner, ${ }^{2}$ H Pavenstädt ${ }^{1}$ Department Nephrology and Rheumatology, University Hospital Muenster, Germany; ${ }^{2}$ Department Immunology, University Erlangen, Germany; ${ }^{3}$ BSRC Fleming, Vari, Greece; ${ }^{4}$ European Institute of Molecular Imaging, University Muenster, Germany; ${ }^{5}$ Div Mol Med Musculoskeletal Tissue, University Hospital Muenster, Germany

\subsection{6/ard.2010.129593j}

The activation of nuclear factor $\kappa \mathrm{B}(\mathrm{NF} \kappa \mathrm{B})$ via $\mathrm{RANK}$ is essential in regulating osteoclastogenesis. Under inflammatory conditions this process is enhanced by cytokines such as tumour necrosis factor $\alpha(\mathrm{TNF} \alpha)$. P62/SOSTM1 directly modulates these pathways through complex formation with TRAF6, aPKCs and ubiquitin. However, the role of p62/SOSTM1 in regulating bone turnover under inflammatory conditions, and specifically the role of the signal transduction domains of p62/ SOSTM1, is unclear.

Mice were generated that carry a shortened but functional mutant of p62 with defective signal transduction domains (p62aa $\Delta 69-251)$ for interbreeding studies with arthritic human TNF transgenic (hTNFtg) mice. The resulting genotypes (wild-type (wt), hTNFtg, p62aa $469-251$ and hTNFtg/ p62aa $\Delta 69-251 / w t$ ) were scored for clinical parameters (paw swelling, grip strength, weight) for 14 weeks. To quantify the extent of inflammation, cartilage degradation and number of osteoclasts, joints of 14 -week-old mice were embedded into paraffin and stained with toluidine-blue and tartrate-resistant acid phosphatase. To identify abnormalities in bone metabolism and bone structure, high resolution micro-CT scanning and positron emission tomography using 18-deoxyfluoroglucose fluoride (FDG-PET) were performed in 14-week-old mice of all genotypes in vivo. In addition, bone marrow derived monocytes (BMDMs) were isolated from all genotypes and osteoclastogenesis was studied using an established osteoclast formation assay. To investigate the underlying signalling pathways, cells were treated with TNF $\alpha$ at different time points and the activation of mitogen-activated protein kinases was studied by western blot analysis.

Histology and in vivo PET/CT studies revealed increased bone metabolism and bone mass in the hind paws, knees and vertebrae of p62aa $\Delta 69-251$ mice, but only minor changes were seen in the number and size of osteoclasts between p62aa $\Delta 69$ 251 and wt animals suggesting that, under physiological conditions, regulatory mechanisms compensate for the lack of the signal transduction domains of p62. Compared with wt cells, however, BMDMs of p62aa $\triangle 69-251$ mice showed a significantly increased osteoclastogenesis, in particular when stimulated with TNF $\alpha$. Crossing of p62aa $\Delta 69-251$ mice with hTNFtg animals resulted in a dramatic increase in the severity of joint damage in the hTNFtg/p62aa $\Delta 69-251 /$ wt mice, as determined clinically by histomorphometry and PET/CT analysis. This was accompanied by an increase in the number and size of osteoclasts in vivo. Western blot analysis of osteoclast lysates revealed increased TNF $\alpha$-induced extracellular signalregulated kinase phosphorylation in cells from p62aa $\Delta 69-251$ compared with wt mice.

Our data suggest that p62 is an important regulator of TNF $\alpha$ mediated joint damage. They indicate that the loss of the TRAF6 and aPKCs binding domains has important consequences for osteoclastogenesis under inflammatory conditions. 